\title{
Le rôle des anaphores dans la mise en place des relations de cohérence dans le discours: l'hypothèse de J.R. Hobbs ${ }^{1}$
}

(Publié dans le Journal of French Language Studies 19(2), 2009, 159-181, Cambridge

University Press ; DOI:10.1017/S0959269509003731)

FRANCIS CORNISH

\author{
CLLE-ERSS, UMR 5263, CNRS et Université de Toulouse-Le Mirail
}

RÉSUMÉ

Pour Hobbs (1979), l'interprétation des anaphoriques inter-phrastiques 'découle' ('falls out') en tant qu'effet secondaire de l'emploi d'une relation de cohérence pour intégrer deux unités de discours. En essayant de mettre au clair la nature des interactions entre relations de cohérence et fonctionnement des anaphores dans la compréhension de textes multipropositionnels, l'article cherche à démontrer que l'hypothèse de Hobbs n'est valable qu'en partie.

D'abord, la référence complète des anaphoriques ne 'découle' pas seulement du choix de telle ou telle relation de cohérence pour intégrer deux unités de discours: elle est essentielle pour la mise en œuvre effective de cette relation. Ensuite, elle sert également à sélectionner l'unité de discours avec laquelle l'unité arrivante va s'intégrer, ainsi qu'à articuler le discours en une structure hiérarchique. Enfin l'intégration des unités de discours n'a pas lieu d'un seul coup, mais peut être étalée sur au moins trois étapes distinctes.

\section{INTRODUCTION}

Tout comme les relations de cohérence, les relations anaphoriques interphrastiques ont comme raison d'être de faciliter la tâche du lecteur ou de l'auditeur - qui est d'intégrer le contenu et la valeur discursive des énoncés arrivants en une structure interprétative plus globale. Comme le disent Zwaan et Rapp (2006: 726), '[c]omprehension requires building connections between [the events being described in the text] and existing representations in memory'. Plus loin (p. 731), les auteurs soutiennent que '...coherence is a direct function of the degree to which comprehenders can connect information they are currently processing, with prior information either in the linguistic stimulus or in memory'.

Ces deux phénomènes (les relations de cohérence et les liens anaphoriques) servent en effet à établir la continuité référentielle sans laquelle une séquence de propositions et de phrases ne formerait pas un

\footnotetext{
1 Je remercie Béatrice Boutin et Michel Aurnague, ainsi que trois relecteurs anonymes de la revue, pour leur relecture attentive de versions antérieures de ce texte.
} 
texte. ${ }^{2}$ L'objectif principal de cet article est de déterminer la nature exacte de leurs interactions.

Après une section présentant l'hypothèse de Hobbs (1979) sur la relation entre la résolution des anaphoriques et la mise en place des relations de cohérence, hypothèse que je me propose de mettre à l'épreuve (\$2), l'article se poursuit en précisant la variété de types d'indices linguistiques qui permettent de reconnaître la pertinence de tel ou tel type de relation de cohérence pour l'intégration de deux unités de discours (\$3). La section 4 est consacrée à une élaboration de définitions d'un sous-ensemble de relations de cohérence, dont la plupart ont été proposées à l'origine par Hobbs (1990); et la section 5 analyse deux courts textes en fonction des indices de reconnaissance détaillés dans la section 3 ainsi que des définitions des relations proposées dans la section 4. Ces analyses sont formulées dans la perspective du compreneur traitant les unités du texte à l'œuvre en temps réel. C'est dans ce cadre que nous verrons la contribution des anaphores dans les phrases et propositions successives dans les textes étudiés. Cornish (sous presse) considère cette problématique à travers les faits de l'anglais, et développe beaucoup plus en détail la question.

\section{L'HYPOTHESE DE HOBBS (1979)}

L'article prend pour point de départ l'hypothèse avancée par Hobbs (1979), qui est la suivante: l'interprétation des anaphoriques inter-phrastiques 'découle' en tant qu'effet secondaire de l'emploi d'une relation de cohérence pour intégrer deux unités de discours. En d'autres termes, une fois qu'une relation de cohérence aura été sélectionnée pour intégrer les propositions logiques et illocutions associables à deux propositions ou phrases dans un texte (qu'elles soient adjacentes l'une de l'autre ou non), au sens où elle pourra être appliquée de façon satisfaisante à ces unités, alors l'interprétation de la ou des expression(s) anaphorique(s) dans la seconde proposition ou phrase sera de ce fait réalisée. Comme le dit Hobbs (1979: 68): 'The solutions to many problems of reference and coreference simply "fall out" in the course of recognizing the coherence relations'. On n'aura donc pas besoin de principes spéciaux pour la résolution des anaphores, en plus de ceux nécessités pour l'établissement d'une relation de cohérence donnée afin d'intégrer le contenu des deux unités de discours.

En guise d'illustration, commençons par ré-analyser l'exemple phare de Hobbs (1979: 78, ex. (3)):

\footnotetext{
${ }^{2}$ Bien entendu, comme me le fait remarquer l'un des relecteurs anonymes, cela ne constitue qu'une condition nécessaire, mais non suffisante, de la cohérence d'un texte. Il faut aussi que les unités discursives créées par intégration successive grâce à une ou plusieurs relation(s) de cohérence locale(s) puissent conjointement satisfaire à la condition (suffisante) de cohérence globale, ou macrocohérence. Celle-ci se reflète dans la possibilité qu'auront ces unités d'être subsumées sous un macrotopique quelconque. Voir à cet égard les analyses proposées par Charolles (2005) de textes à première vue incohérents.
} 
(1) John can open Bill's safe. He knows the combination.

Dans (1), l'un comme l'autre des deux référents humains mâles évoqués dans la phrase initiale pourra en principe être repris via le pronom sujet masculin au singulier he dans la seconde; de plus, la propriété qui en est prédiquée consistant à « connaître le chiffre de combinaison de la serrure d'un coffre-fort particulier » pourra s'appliquer de façon plausible à chacun des référents à l'œuvre (à 'Bill', puisque le coffre-fort en question lui appartient, et à 'John', puisque c'est ce qu'affirme la phrase initiale). Pourtant, c'est seulement lorsque le pronom he dans la seconde phrase est compris comme renvoyant à 'John' que la proposition logique qu'elle exprime pourra être interprétée comme fournissant un Indice ou une Preuve ('Evidence') étayant l'Assertion ('Claim') faite à propos de John via la première. La phrase initiale en tant qu'énoncé peut s'analyser comme exprimant une structure informationnelle thétique (où l'information qu'elle véhicule est présentée comme 'toute nouvelle'), et la seconde comme articulation 'catégorique' (à topique-commentaire), reprenant comme topique le référent le plus proéminent évoqué via la première ('John'). Le fait d'interpréter he comme renvoyant à 'Bill' dans (1) ne permettrait pas à la proposition logique ainsi créée de fournir une information supplémentaire à propos de 'John'. De plus, comme les pronoms de $3^{\text {eme }}$ personne inaccentués sont spécialisés dans la reprise de référents hautement topicaux et présupposés, l'interprétation de he ici en fonction du référent du $\mathrm{SN}$ génitif Bill('s), déterminant nominal possessif - donc en position périphérique dans la phrase initiale_-, serait de toute façon difficile. Du coup, la continuité de la situation évoquée via l'assertion de la phrase initiale ne serait pas assurée. ${ }^{3}$

A proprement parler, plutôt que de dire que la résolution du pronom he 'découle' simplement de la mise en place d'une relation de cohérence intégrant les deux unités, comme le soutient Hobbs (1979), cela est en fait un pré-requis pour la mise en œuvre d'une relation appropriée; mais il est clair que les deux procédures fonctionnent en symbiose l'une avec l'autre (cf. Cornish (sous presse)).

Hobbs, pour sa part, analyse la seconde phrase de (1) comme étant dans une relation d'Élaboration par rapport à la proposition évoquée via la première (au sens où c'est la même proposition qui est inférée dans chaque cas, moyennant l'emploi de mots différents): ${ }^{4}$ car si $\mathrm{X}$ sait ouvrir $\mathrm{Y}$, où $\mathrm{Y}$ est un coffre-fort, alors $X$ connaît le numéro de combinaison qui permet d'ouvrir Y (les coffres-fort étant habituellement sécurisés de cette façon).

\footnotetext{
${ }^{3}$ Le discours construit ainsi serait de ce fait singulièrement peu informatif, de toute façon.

${ }^{4}$ Voir la contribution de Kleiber et Vassiliadou (ce numéro) pour un examen approfondi de cette relation, notamment en ce qui concerne sa conception chez Hobbs.
} 
Dit autrement, cette proposition est impliquée à la fois par la phrase initiale et par la seconde. Cela est clairement le cas ici; mais cette relation plus fondamentale reliant les deux propositions peut (en fait, doit) être renforcée en interprétant la phrase initiale comme effectuant une Assertion particulière (la présence de l'auxiliaire modal can sert à motiver cette interprétation), et en interprétant la seconde comme fournissant à l'allocutaire ou au lecteur une raison de croire à cette Assertion. Si tout ce qui était en jeu dans la compréhension du texte (1) revenait à une simple paraphrase par la seconde phrase de la première, alors cela enfreindrait un principe fondamental de la communication: 'Ne dites pas à votre allocutaire ce qu'il sait déjà - à moins qu'il existe une bonne raison de le faire' (ce serait le cas ici si la seconde phrase était comprise comme fournissant une preuve de l'assertion effectuée via la première - le locuteur/scripteur ressentant le besoin de persuader l'allocutaire/lecteur de la plausibilité de son assertion via la première).

Un argument supplémentaire en faveur de la pertinence de la relation Assertion-Indice plutôt qu'une simple 'Élaboration' dans le cas de (1), est le fait que le connecteur after all 'après tout' pourra être inséré de façon cohérente entre les deux phrases —sans affecter d'aucune façon l'interprétation originelle.

\section{LA RECONNAISSANCE DES RELATIONS DE COHÉRENCE: QUELQUES TYPES D'INDICES PERTINENTS}

Il y a plusieurs types d'indices linguistiques permettant de déterminer la pertinence d'application de telle ou telle relation de cohérence. Cependant, ces indices ne sont pas dans un rapport biunivoque avec telle ou telle relation de cohérence: ${ }^{5}$ toutefois, la convergence ou la prépondérance d'indices de types différents peut bien permettre de conclure à l'applicabilité d'une relation quelconque. On l'a vu dans l'exemple (1), où la possibilité d'insertion du connecteur after all entre les deux phrases, la présence de l'auxiliaire modal can au temps présent à valeur stative (prédicateur-hôte 'can open') ainsi que le caractère thétique de cette phrase en tant qu'énoncé, œuvrent ensemble pour lui attribuer la valeur d'une Assertion ('Claim'); et où le lien anaphorique entre le pronom sujet he et le sujet de la phrase initiale John (ainsi que l'anaphore associative reliant Bill's safe et le $\mathrm{SN}$ objet direct the combination) contribuent à donner à la seconde phrase le statut d'un énoncé catégorique à topique-commentaire, prédiquant une propriété (une capacité particulière) à propos du référent 'John'. Le temps du verbe de cette seconde phrase est également le présent, qui est aussi à valeur stative grâce à l'Aktionsart statif du prédicateur 'know'. Il n'y a donc aucune progression temporelle impliquée ici, la situation désignée

\footnotetext{
${ }^{5}$ Ceci est bien entendu la raison pour laquelle Mann et Thompson (1988) avaient rejeté le recours aux indices 'textuels' dans leurs définitions de telles relations.
} 
par la seconde phrase étant la même que celle évoquée par la première (cf. aussi Dowty, 1986). Ainsi, le statut d'Indice ou de Preuve de l'énoncé qui lui correspond peut être fondé ici.

Les indices heuristiques pertinents sont donc le type de connecteur utilisé ou insérable pour relier les deux unités; ${ }^{6}$ les relations temporelles et aspectuelles existant entre les propositions concernées: temps du verbe, adverbiaux temporels; les relations lexicales qui peuvent exister entre les prédicateurs des propositions à relier (relations d'hyponymie, de synonymie, de méronymie, d'antonymie ou la relation converse) ${ }^{7}-$ mais plus généralement, la relation entre ce qui est prédiqué dans chacune des unités à relier; l'Aktionsart (aspect lexical) des prédicateurs verbaux des deux propositions à relier: statuts d'état, d'activité, d'achèvement ou d'accomplissement, et partant, la structure événementielle ou 'aspectuelle' des deux propositions à l'œuvre dans leur ensemble. ${ }^{8}$ La combinaison des structures informationnelles particulières associées à chaque unité en tant qu'énoncé (énoncé thétique suivi d'énoncé catégorique - à topiquecommentaire - dans (1), par exemple) peut également favoriser l'invocation de tel type de relation de cohérence plutôt que tel autre pour intégrer les deux unités. Voir Luscher (1998) pour une considération des influences mutuelles exercées par l'emploi de certains connecteurs (donc, parce que et zéro - autrement dit, une situation de parataxe entre deux phrases adjacentes), des pronoms personnels de reprise dans la seconde phrase, et du choix de temps verbaux (surtout le passé composé et le plusque-parfait) dans chacune d'entre elles. Voici donc les indices pertinents (Tableau 1):

Aucun de ces critères, pris individuellement, ne serait suffisant pour fonder l'existence de telle ou telle relation de cohérence: c'est plutôt leur interaction qui permettrait éventuellement de le faire. Voir Cornish (2006) pour une présentation plus développée des indices de reconnaissance des relations de cohérence en discours.

\footnotetext{
${ }^{6}$ Mais il faut bien entendu tenir compte du fait que tel connecteur pourra avoir plus d'une valeur cohésive. Voir Rossari (2000) pour une discussion approfondie du fonctionnement discursif des connecteurs, ainsi que Sanders et Noordman (2000) et Webber et al. (2003). Voir ici-même les analyses des connecteurs d'abord par Bras et Le Draoulec, puis de notamment par Vergez-Couret.

7 Voir Murphy (2003) pour une présentation récente basée sur les faits de l'anglais.

8 Voir Rothstein (2004) pour une discussion éclairante.
} 


\begin{tabular}{|c|c|}
\hline Type d'indice de reconnaissance & Exemples d'indices \\
\hline $\begin{array}{l}\text { 1) La possibilité d'insérer entre chaque paire de } \\
\text { phrases indépendantes un connecteur d'un certain } \\
\text { type. L'interprétation avec le connecteur doit être } \\
\text { identique à celle qui prévaut sans le connecteur. }\end{array}$ & $\begin{array}{l}\text { Mais pour la relation Contraste ou bien Concession; } \\
\text { En effet et après tout pour la relation Assertion-Indice; } \\
\text { Notamment ou en particulier pour la relation Élaboration; } \\
\text { Donc et de ce fait pour la relation Cause-Conséquence; } \\
\text { Parce que/Car pour la relation Explication; } \\
\text { De même ou et pour la relation Parallèle; } \\
\text { Puis pour la relation Narration; } \\
\text { Certes/Si /Bien que pour la relation Concession. }\end{array}$ \\
\hline $\begin{array}{l}\text { 2) L'existence de relations lexicales ou } \\
\text { sémantiques/encyclopédiques entre les prédicateurs } \\
\text { des deux phrases. }\end{array}$ & 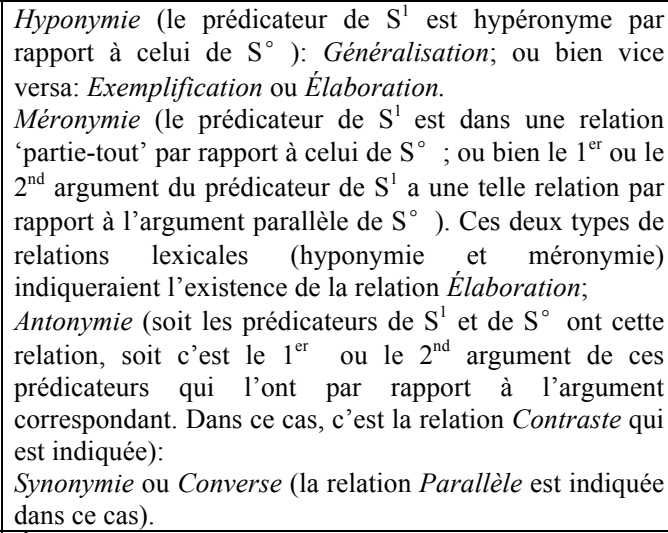 \\
\hline $\begin{array}{l}\text { 3) L'Aktionsart du prédicateur de chaque phrase ou } \\
\text { proposition, et partant, la structure événementielle } \\
\text { des paires de phrases impliquées. }\end{array}$ & $\begin{array}{l}\text { État, Processus/Activité, Achèvement, Accomplissement. } \\
\text { Pour que la relation Narration puisse être pertinente, il } \\
\text { faudra qu'au moins la } \mathrm{S}^{1} \text { corresponde à l'une des trois } \\
\text { dernières valeurs. Pour la relation Élaboration, la } \\
\text { structure événementielle de } \mathrm{S}^{1} \text { peut être celle d'un état, et } \\
\text { pour Résultat, elle doit l'être. }\end{array}$ \\
\hline $\begin{array}{l}\text { 4) Les temps verbaux } \\
\text { phrases successives du texte. }\end{array}$ & $\begin{array}{l}\text { Si le temps du verbe (principal) de la } \mathrm{S}^{1} \text { est de nature à } \\
\text { faire avancer le temps réel désigné par } \mathrm{S}^{\circ} \text { (passé } \\
\text { composé, passé simple, 'présent de narration,', par rapport } \\
\text { à l'imparfait, au plus-que-parfait ou au présent tout court), } \\
\text { alors c'est la relation Narration qui est indiquée (mais } \\
\text { cette possibilité est contrainte par le type d'Aktionsart du } \\
\text { prédicateur concerné). Le plus-que-parfait ainsi que } \\
\text { l'imparfait signaleraient l'existence des relations } \\
\text { 'subordonnantes' Élaboration, Explication ou Assertion- } \\
\text { Indice. }\end{array}$ \\
\hline $\begin{array}{l}\text { 5) Les relations anaphoriques (orientant vers tel ou } \\
\text { tel point d'attachement de la proposition contextuelle, } \\
\text { puis, surtout, créant l'ancrage référentiel indispensable } \\
\text { qui permettra à la RC choisie d'intégrer les deux unités } \\
\text { en jeu). La présence d'un anaphorique dans une clause } \\
\text { indiquerait la subordination de la clause hôte, au plan } \\
\text { pragmatico-discursif, par rapport à la clause qui permet } \\
\text { de résoudre le ou les anaphorique(s). }\end{array}$ & $\begin{array}{l}\text { Éléments nuls, pronoms clitiques de } 3^{\text {ème }} \text { personne, } \\
\text { pronoms démonstratifs, SN définis réduits, SN } \\
\text { démonstratifs réduits, SN possessifs, ellipses. Ces } \\
\text { marqueurs opèrent en fonction de leurs propriétés } \\
\text { sémantiques et indexicales propres, en liaison avec la } \\
\text { fonction orientationnelle du contenu prédicatif de leur } \\
\text { proposition hôte. }\end{array}$ \\
\hline
\end{tabular}

Tableau 1. Indices de reconnaissance des relations de cohérence

\section{Voici en illustration un exemple français d'un résumé de film, extrait} de notre premier corpus: 
(2) 'L'ÎLE DE ROBINSON Téléfilm ( $\cdots)$

$\left[\mathrm{UD}^{\circ}{ }_{\text {LOC-TPS }}^{9} \mathrm{Au}\right.$ XVIIIè siècle], $\left[\mathrm{UD}^{1}{ }_{\mathrm{EV}}\right.$ le propriétaire d'une plantation s'oppose, $\left[\mathrm{UD}_{\mathrm{EV}}^{2}\right.$ lors d'une traversée maritime], au capitaine du bateau]. [ $\mathrm{UD}_{\mathrm{EV}}^{3} \mathrm{Il}$ est abandonné sur une île.]' Le Monde Supplément Radio-Télévision 22-28/12/03, p. 8.

Suite à l'occurrence cadrative d'un 'adverb[ial] de localisation directe' (au XVIIIè siècle - $\mathrm{UD}^{\circ}{ }_{\text {LOC-TPS }}$, selon la taxinomie de Borillo $(1998: 132,136)$ ayant portée sur l'ensemble du texte, l'UD $^{1}$ présente la situation de base qui forme la trame du film: le temps présent est un présent de narration (cette valeur est en partie due au genre du texte, un résumé de film dans lequel on s'attend au récit d'une histoire, en partie à l'Aktionsart du verbe s'opposer, et au SP événementiel en apposition lors d'une traversée maritime). ${ }^{10} \mathrm{Ce}$ $\mathrm{SP}\left(\mathrm{UD}^{2}\right)$, de par la proposition nominalisée qu'il contient, correspond à une unité de discours, marquée à la fois par la préposition temporelle lors (de) et par l'Aktionsart d'accomplissement représenté dans le $\mathrm{N}^{\prime}$ traversée

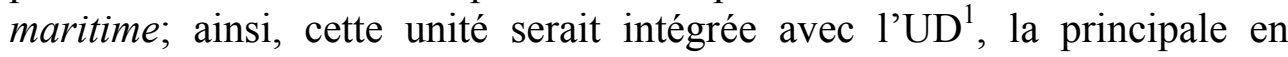
termes textuels, en fonction de la relation Circonstance. Le SN défini au sein du SP du bateau dans l'UD ${ }^{1}$ reprend un référent introduit implicitement dans le discours en amont via l'interprétation du segment lors d'une traversée maritime dans $1^{\prime} \mathrm{UD}^{2}$, qui se trouve en incise. Cette inférence serait à la fois 'élaborative' et 'connective', selon Zwaan et Rapp (2006: 735). ${ }^{11}$ L'unité composée $\left[\mathrm{UD}^{1}+\mathrm{UD}^{2}\right]$ correspond à un énoncé à caractère thétique.

Le passif du verbe abandonner au temps présent (toujours de narration) de l'UD ${ }^{3}$ qui suit met l'accent sur l'état résultant d'une action précédente. Grâce au fonctionnement du sujet pronominal référentiel $i l$,

\footnotetext{
9 Chaque unité minimale dégagée du texte (correspondant au plan textuel à une proposition finie ou non-finie, principale ou indépendante, coordonnée ou subordonnée, ou bien nominalisée - voire à un SN ou SP 'cadratif') est annotée pour sa structure événementielle, ou bien son rôle sémantique (LOCTPS = 'Localisation temporelle'). J'emploie pour cela la notation proposée par Smith (2003) (mentions en indice 'EV' pour 'événement' ou 'ET' pour 'état'). Les symboles préfixés ' $\mathrm{UD}^{\circ}$ ', ' $\mathrm{UD}^{1}$ ', etc. sont employés pour représenter les unités minimales du texte, correspondant aux actes de discours qu'elles permettent de réaliser. 'UD' symbolise 'Unité de Discours'. Hobbs (1990), lui, les envisage comme unités phrastiques, notées ' $\mathrm{S}{ }^{\circ}$, , ' $\mathrm{S}$ ', etc. Voir Roulet (2002) pour des arguments motivant la réalisation d'une unité de discours par des SN et SP tels que le SP adverbial antéposé dans (2). Les relatives restrictives et complétives compléments, par contre, ne correspondent pas à des unités de discours susceptibles de renfermer un acte discursif. Voir Cornish (sous presse) pour de plus amples explications de ce système de notation.

10 Voir Adam (2000) pour des analyses éclairantes des valeurs (non strictement temporelles) d'un éventail de temps verbaux en français employés dans des résumés de films (comme le texte (2)) et des articles de presse de faits divers, entre autres. Voir aussi Mellet (1998) pour une approche 'aspectuelle' du présent de narrration.

11 Une inférence 'élaborative' implique une activation de connaissances qui rehaussent la représentation mentale de la situation évoquée, mais dont on n'a pas besoin pour intégrer les deux assertions; alors qu'une inférence 'connective' constituerait un moyen de relier deux assertions entre elles (Zwaan et Rapp, 2006: 735).
} 
cette phrase va naturellement revêtir un statut informationnel catégorique (topique-commentaire). Le contenu de cette seconde phrase indépendante sera donc intégré à celui de l'unité composée $\left[\mathrm{UD}^{1}+\mathrm{UD}^{2}\right]$ en vertu de la relation Résultat Intentionnel (terme de Mann et Thompson, 1988), ce en fonction de notre connaissance du monde (surtout des mœurs maritimes de l'époque): à savoir, que le capitaine d'un bateau ${ }^{12} \mathrm{a}$ en principe le droit de régir le comportement des personnes à bord de son navire. Etant donné l'existence d'une dispute entre celui-ci et un passager sur son bateau ('le propriétaire d'une plantation') évoquée par l'UD', conjointement au pouvoir du capitaine sur les passagers à bord de son bateau, il est plus probable que ce sera le propriétaire de la plantation que le capitaine du bateau qui sera 'abandonné sur une île', suite à cette altercation.

Pour ce qui concerne le test de l'insertion de tel ou tel type de connecteur entre les phrases correspondant aux unités de discours à relier, dans (2) nous pouvons proposer que le connecteur de ce fait marquant la relation de Résultat, aurait pu être antéposé à $1^{\prime} U^{3}$. Cette relation serait chapeautée ici par celle de Problème-Solution (type de relation reconnu par Mann et Thompson, 1988), étant donné que l'UD $^{1}$ fait état d'une situation conflictuelle (...s'oppose...), donc 'problématique', situation à laquelle l'abandon du propriétaire de la plantation sur une île pourrait constituer une 'solution', aux yeux du capitaine. Le même raisonnement concernant la résolution du pronom anaphorique il sujet de $1^{\prime} U^{3}$ pour fonder la pertinence de Résultat Intentionnel s'appliquerait dans ce cas (voir plus bas).

La mise en place des relations Résultat Intentionnel et ProblèmeSolution afin d'intégrer les deux unités exige donc que le pronom sujet il soit résolu en ciblant le propriétaire de la plantation, plutôt que le capitaine. En principe (hors contexte particulier), cependant, ce pronom masculin singulier pourrait être interprété comme renvoyant au 'capitaine', voire même au 'bateau'. ${ }^{13}$ Après tout, et un bateau et un capitaine de navire peuvent fort bien 'être abandonnés sur une île'. Mais dans l'un comme l'autre de ces deux cas, les relations Résultat Intentionnel et ProblèmeSolution n'auraient que peu de justification comme moyens d'intégrer les deux unités à l'œuvre ici. Voir le même point relevé à propos de l'analyse de l'exemple anglais (1) ci-dessus.

\footnotetext{
12 L'agent implicite du procès passif en $\mathrm{UD}^{3}$ va donc être 'le capitaine du bateau'.

13 Dans ce dernier cas, toutefois, cette interprétation serait plus difficile, étant donné que le SN le bateau est enchâssé dans un SP, lui-même complément du N capitaine. Comme dans le cas de figure où he reprendrait le référent 'Bill' dans l'exemple anglais (1), le référent de ce $\mathrm{SN}$ ne jouit que d'un degré peu élevé de topicalité dans ce contexte.
} 
La Figure 1 ci-dessous représenterait la structure du texte (2) d'après la Rhetorical Structure Theory (RST) de Mann et Thompson (1988), ${ }^{14}$ et la Figure 1a selon l'approche de Hobbs (1990). À noter que c'est la représentation hobbsienne qui montre de façon plus explicite la hiérarchisation des rattachements entre les unités en jeu ici (le symbole UD ${ }^{1}$ en gras dans cette dernière Figure représente l'unité dominante par rapport à l'unité qui lui est rattachée). Comme Mann et Thompson excluent la possibilité que plus d'une relation s'applique pour relier deux unités simultanément, les deux relations en question sont présentées ici comme alternativement pertinentes pour intégrer l'unité 3 à l'unité composée $2+1$ dans la Figure 1; mais comme Hobbs, lui, l'admet, Problème-Solution est superposée à Cause-Conséquence (l'équivalent hobbsien de Résultat Intentionnel chez Mann et Thompson) dans la Figure 1a - sa position supérieure indiquant qu'elle est dominante par rapport à celle-ci (ce système de représentation est de mon cru). Voir Webber et al. (2003) et Egg et Redeker (2008) pour deux types de représentation arborescente semblables (mais non identiques) de la structure de discours des textes.

Circonstance 1-3

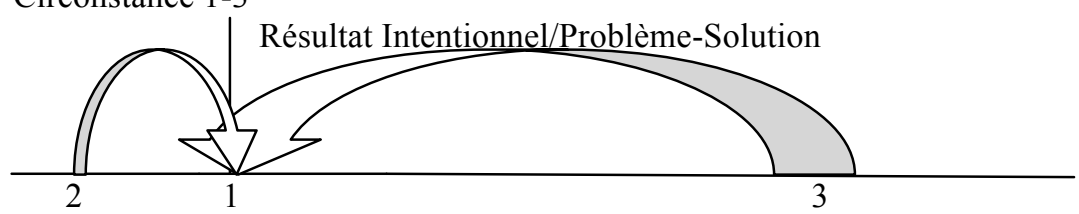

Figure 1. Diagramme RST pour le texte (2) (FC)

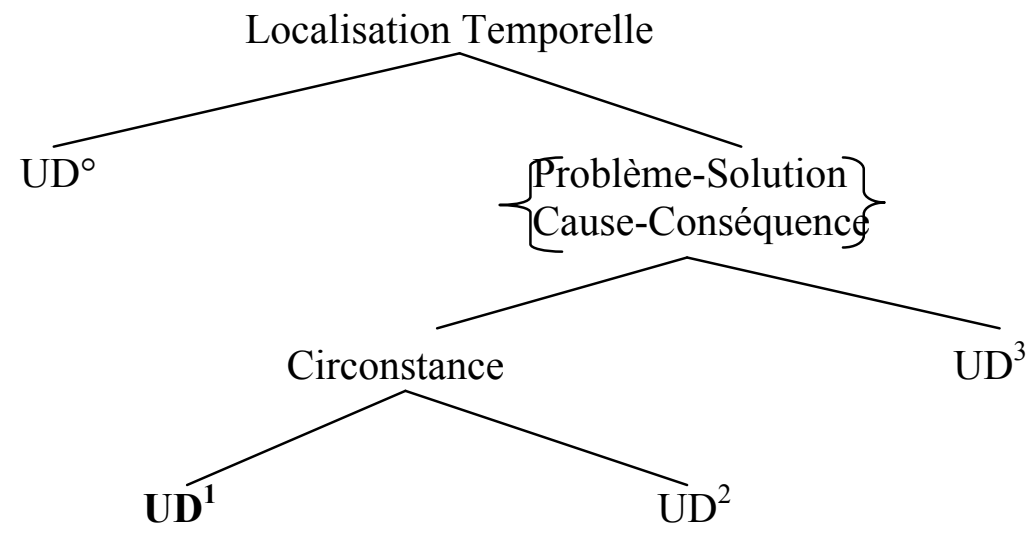

Figure 1a. Représentation (modifiée) d'après Hobbs (1990) de la structure de discours associée au texte (2). Nota: l'UD en caractères gras dans cette Figure est l'unité dominante dans la relation concernée.

\footnotetext{
14 Sans toutefois tenir compte de l'unité UD ${ }^{\circ}$ (réalisée par le SP cadratif de localisation temporelle), car il ne semble pas y avoir moyen de la représenter selon le modèle RST. Cette unité est en revanche représentée dans la Fig. 1a, selon le modèle de Hobbs.
} 


\section{LA FORMULATION DES RELATIONS DE COHÉRENCE}

Dans les exemples présentés jusqu'à présent, nous avons vu les relations de cohérence suivantes: Élaboration, Assertion-Indice, Circonstance, Résultat/Cause-Conséquence et Problème-Solution. Nous avons également vu que certaines relations peuvent s'appliquer simultanément pour intégrer deux unités de discours (Élaboration et Assertion-Indice dans le texte (1), Résultat et Problème-Solution dans le texte (2), puis Occasion et Explication en (3) (section 5 plus bas)). Ceci est possible, intuitivement parlant, puisqu'Assertion-Indice, par exemple, implique normalement une 'Élaboration' de l'état de choses évoqué par la première unité à l'œuvre, et puisque Problème-Solution implique que la 'Solution' au 'Problème' posé en constitue le 'Résultat', en quelque sorte (à condition, bien entendu, que ce 'Résultat' ait été intentionnel - comme dans le texte (2) -, et non accidentel). Mais il faut aussi que la structure interne de chacune des relations superposées soit compatible - plus précisément, que la partie pragmatiquement dominante ou 'subordonnante' dans la relation inférieure corresponde à celle de la relation supérieure, lorsque les relations sont effectivement 'subordonnantes' (voir plus bas) - donc, que les relations concernées aient une structure interne parallèle. C'est-à-dire qu'il ne faut pas qu'une relation superposée à une autre ait une structure interne qui ne soit pas parallèle à celle de la relation 'subsumée'.

\subsection{La structure interne des relations de cohérence}

Comme le point de départ de cet article a été l'hypothèse de Hobbs (1979) sur la relation univoque entre relations de cohérence et résolution des anaphores, il a semblé logique de prendre pour base ses propres conceptions de telles relations. On les modifiera en fonction d'un examen critique de définitions théoriques comparables (notamment, celles de Mann et Thompson, de Sanders, de Kehler, et d'Asher et Lascarides), ainsi que d'analyses des données de nos corpus. Chez Hobbs, les principes fondant les relations de cohérence se ramènent pour l'essentiel à trois types principaux : ceux de 'Causalité', de 'Fond-Figure', et d' Expansion'. Dans le tableau 2, j'essaie de classifier les différentes sous-catégories de relations reconnues par Hobbs (ainsi que quatre de plus) sous ces trois rubriques. Parfois, j'ai modifié une définition (Élaboration, Explication), ajouté ma propre définition (Cause-Conséquence/Résultat, Circonstance, Assertion-Indice), ou changé la classification de Hobbs (Explication, transférée de 'FondFigure' à 'Causales'). Voir Cornish (sous presse) pour de plus amples explications. 
Tableau 2. Définitions d'un sous-ensemble de Relations de Cohérence selon, et d'après, Hobbs (1990, ch. 5)

\begin{tabular}{|c|c|c|}
\hline Relations Causales & Relations Fond-Figure & Relations d'Expansion \\
\hline $\begin{array}{l}\text { Occasion: 1) Inférer un } \\
\text { changement d'état de l'assertion } \\
\text { de } S^{\circ} \text {, dont l'état final peut être } \\
\text { inféré de } S^{1} .2 \text { 2) Inférer un } \\
\text { changement d'état de l'assertion } \\
\text { de } S^{1} \text {, dont l'état initial peut être } \\
\text { inféré de } S^{\circ} \text {. (Hobbs, 1990: } 87 \text { ) }\end{array}$ & $\begin{array}{l}\text { Fond-Figure: Inférer de } S^{\circ} \text { une } \\
\text { description d'un système } \\
\text { d'entités et de relations, et } \\
\text { inférer de } S^{1} \text { qu'une certaine } \\
\text { entité est située ou bien se } \\
\text { déplace dans ce système en tant } \\
\text { qu'arrière-fond. (Hobbs, 1990: } \\
91 \text { ) }\end{array}$ & $\begin{array}{l}\text { Parallèle : Inférer } p\left(a_{1}, a_{2}, \ldots\right) \\
\text { de l'assertion de } \mathrm{S}^{\circ} \text { et } p\left(b_{1} \text {, }\right. \\
\left.b_{2}, \ldots\right) \text { de l'assertion de } \mathrm{S}^{1} \text {, où } a_{i} \\
\text { et } b_{i} \text { sont semblables, pour tout } \\
i .(\text { Hobbs, 1990: } 93)\end{array}$ \\
\hline $\begin{array}{l}\text { Cause-Conséquence } \\
\text { [“Résultat"]: cas particulier de la } \\
\text { relation Occasion (sa version } \\
\text { 'forte'). Inférer que l'état ou } \\
\text { l'événement asserté via } \mathrm{S}^{\circ} \text { cause } \\
\text { ou pourrait causer l'état ou } \\
\text { l'événement asserté via } \mathrm{S}^{1} \text {. (FC: } \\
\text { définition basée sur la définition } \\
\text { que donne Hobbs de la relation } \\
\text { Explication - voir la cellule } 3 \text {, } \\
\text { cette colonne). }\end{array}$ & 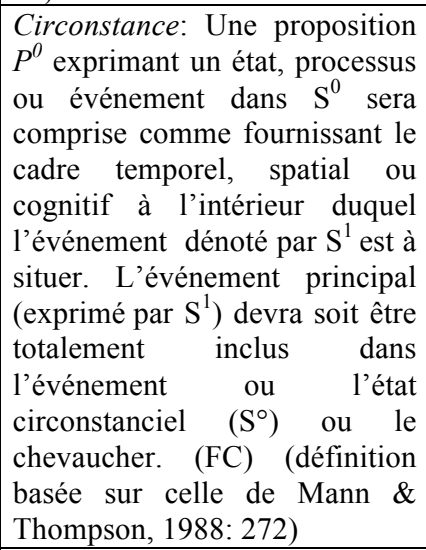 & 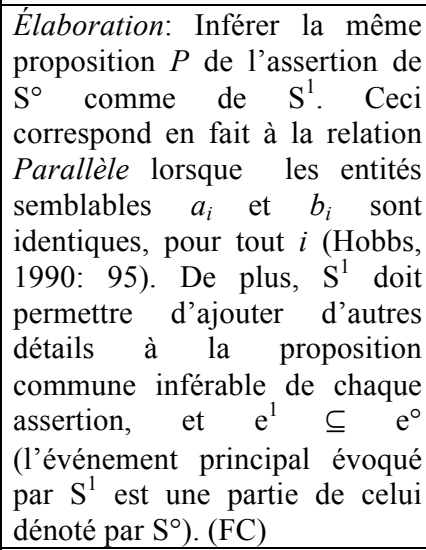 \\
\hline \multirow[t]{2}{*}{ 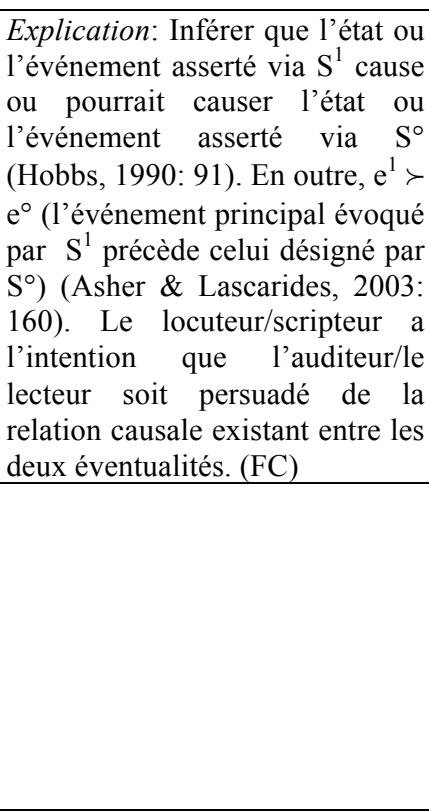 } & & $\begin{array}{l}\text { Contraste: 1) Inférer } p(a) \text { de } \\
\text { l'assertion de } \mathrm{S}^{\circ} \text { et } \neg p(b) \text { de } \\
\text { l'assertion de } \mathrm{S}^{1} \text {, où } a \text { et } b \text { sont } \\
\text { semblables. 2) Inférer } p(a) \text { de } \\
\text { l'assertion de } \mathrm{S}^{\circ} \text { et } p(b) \text { de } \\
\text { l'assertion de } \mathrm{S}^{1} \text {, où il existe } \\
\text { quelque propriété } q \text { telle que } \\
q(a) \text { et } \neg q(b) \text {. (Hobbs, 1990: } \\
99)\end{array}$ \\
\hline & & 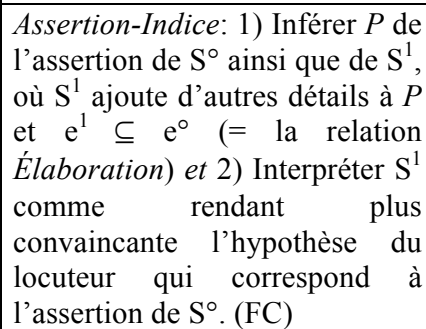 \\
\hline
\end{tabular}

Tout d'abord, dans la première colonne du Tableau, les relations Occasion et Cause-Conséquence seraient basées, selon Hobbs (1990: ch. 5), sur la causalité. La relation Occasion correspond à la préparation par l'événement évoqué dans la proposition initiale (ou seconde) de celui dénoté par la proposition qui la suit (ou la précède) (voir la cellule $1,1^{\text {ère }}$ colonne $\mathrm{du}$ Tableau 2). Voir comme illustration la relation initiale reliant l' $^{\prime} \mathrm{UD}^{\circ}$ et 
$1^{\prime} \mathrm{UD}^{1}$ dans le texte (3) à la section $5 .^{15}$

J'ai formulé la relation Cause-Conséquence (Résultat) en termes de la définition que fournit Hobbs de la relation Explication - où Cause et Effet sont inversés: voir les cellules 2 et 3 de la $1^{\text {ère }}$ colonne du Tableau 2, respectivement. Comme exemple de Résultat, voir la (seconde) relation intégrant $1^{\prime} \mathrm{UD}^{3}$ et l'unité composite $\left[\mathrm{UD}^{1}+\mathrm{UD}^{2}\right]$ dans le texte (2). Mais Explication suppose que le locuteur/scripteur a l'intention que l'éventualité causatrice 'explique' celle qui est causée pour l'allocutaire/le lecteur. Il ne s'agit pas simplement d'une relation 'objective' (sémantique) entre deux éventualités (comme ce serait le cas avec la relation Résultat). En plus de cette précision, j'ai ajouté à la définition la contrainte temporelle imposée sur la relation Explication par Asher et Lascarides (2003: 160): à savoir, que l'événement causateur précède normalement l'événement causé. Voir comme illustration la relation 'superordonnée' reliant l'UD $^{\circ}$ et l'unité composée $\left[\mathrm{UD}^{1}\left[+\mathrm{UD}^{2}\left[+\mathrm{UD}^{3}\right]\right]\right]$ dans (3) ci-dessous (section 5). Comme le note Kehler (2004), les relations causales impliquent comme arguments les propositions logiques exprimées par les phrases ou propositions reliées.

Dans la deuxième colonne, les relations 'Fond-Figure' (dont Hobbs ne cite que la relation Explication - que j'ai placée sous la rubrique 'Relations Causales' dans le Tableau 2 - en plus de cette relation canonique) sont censées relier un segment de discours au stock (activé) de connaissances préalables chez l'allocutaire. La relation reliant l'UD' à l'UD' ${ }^{2}$ du texte (4) ci-dessous (section 5) illustre la relation canonique Fond-Figure - même si la présentation du 'Fond' suit celle de la 'Figure', au plan textuel. ${ }^{16}$

Un autre cas typique d'une relation 'Fond-Figure' serait celle de Circonstance (essentiellement définie par Mann et Thompson, 1988: 272 comme la mise en place par le satellite d'un 'framework in the subject matter within which $\mathrm{R}$ [le lecteur] is intended to interpret the situation presented in $\mathrm{N}$ [le noyau]'). Mais cette définition semble caractériser plus généralement la relation Fond-Figure plutôt que celle, plus spécifique, de Circonstance. Voir la $2^{\mathrm{e}}$ colonne du Tableau $2\left(2^{\mathrm{e}}\right.$ cellule) pour la définition à inflexion plus temporelle que je propose à la Hobbs, en partie inspirée par celle de Mann et Thompson. Une illustration se trouve dans le SP temporel $\mathrm{UD}^{2}$ lors d'une traversée maritime dans le texte (2) ci-dessus.

Enfin, les 'Relations d'Expansion' (voir la $3^{\mathrm{e}}$ colonne du Tableau 2: ici, celles de Parallèle, d'Élaboration et de Contraste - auxquelles j'ai ajouté celle d'Assertion-Indice) seraient des instanciations du principe général de 'Ressemblance'. Pour Hobbs, les 'Relations d'Expansion' sont

\footnotetext{
15 Kehler (2004) place cette relation dans la catégorie 'Contiguité', qui correspond aux relations 'Fond-Figure' chez Hobbs. Ceci parce que l'état final de l'état de choses antérieur est l'état initial de l'état de choses postérieur.

${ }^{16}$ Ici, l'UD ${ }^{2}$ représente le 'Fond' (l'arrière-plan) en fonction duquel l'UD ${ }^{1}$ correspondra à la 'Figure' (le premier plan de la situation évoquée). Voir ici-même la contribution de Prévot, Asher et Vieu en ce qui concerne la relation d'Arrière-Plan dans le modèle SDRT.
} 
des relations qui étendent le discours in situ, plutôt que de le faire avancer ou de développer son arrière-plan. Elles impliquent toutes des relations inférentielles entre les segments du co-texte, et servent à faciliter les processus inférentiels du compreneur.

Quant à la définition de Hobbs de la relation Élaboration (voir la $2^{\mathrm{e}}$ cellule de la $3^{\mathrm{e}}$ colonne du Tableau 2), j'ai ajouté la condition selon laquelle $\mathrm{S}^{1}$, la proposition élaborante, devra apporter des informations à la proposition commune inférée de chaque phrase - car sinon, la définition ne reviendrait à rien de plus qu'une simple relation de 'Paraphrase' (voir l'exemple de Hobbs, 1979 donné comme (1) ci-dessus, sous son analyse en termes d' Élaboration'). ${ }^{17}$ De plus, l'événement principal (symbolisé $\mathrm{e}^{1}$ ) évoqué via $\mathrm{S}^{1}$, l'unité élaborante, devra être interprétable comme faisant partie intégrante de celui dénoté par $\mathrm{S}^{\circ}\left(\mathrm{e}^{\circ}\right)$, l'unité élaborée (Asher et Lascarides, 2003; Fabricius-Hansen et Behrens, 2001). Voir comme exemple canonique d'Élaboration le mode d'intégration des unités composées $\left[\mathrm{UD}^{4}+\mathrm{UD}^{5}\right]$ et $\left[\mathrm{UD}^{\circ}+\left[\mathrm{UD}^{1}+\left[\mathrm{UD}^{2}+\left[\mathrm{UD}^{3}\right]\right]\right]\right]$ dans le texte (3) plus bas (section 5).

Comme le fait observer Kehler (2004), les relations d'Expansion' ou de 'Ressemblance' impliquent toutes une relation entre des arguments dénotant des entités, et non (directement) des propositions entières, comme dans le cas des relations causales ou à Fond-Figure/Contiguïté: voir les structures sémantiques de chacune des relations présentées dans la $3^{\mathrm{e}}$ colonne du Tableau 2.

Par ailleurs, en observant des données attestées plutôt qu'uniquement des données fabriquées pour la démonstration, il semblerait que les définitions des relations de cohérence ne devraient pas être conçues en termes absolus, 'catégoriques' (cf. le commentaire de Fabricius-Hansen \& Behrens, 2001: 2 sur la relation Élaboration): plutôt, elles correspondraient à des prototypes, où les relations observées existant entre propositions ou arguments dénotant des entités inférables à partir des textes, conjointement à des contextes donnés, pourront se rapprocher plus ou moins étroitement de la caractérisation 'noyau' (Hobbs, 1979: 73 lui-même est de cet avis).

Ces relations peuvent être subdivisées en deux macro-catégories qui sont orthogonales par rapport aux trois catégories reconnues comme fondant les différents types de relations de cohérence (Hobbs, 1990: 104): à savoir, relations coordonnantes et relations subordonnantes. ${ }^{18}$ Voir aussi Asher et Vieu (2005) pour une conception adaptée au modèle SDRT de la structure de discours des textes. Le critère sur lequel Hobbs se base pour classifier les relations ainsi est celui qui correspond à la nature de ce qui est asserté dans les deux propositions ou phrases à l'œuvre: dans le cas des relations

\footnotetext{
17 Voir ici-même l'article de Kleiber et Vassiliadou pour une discussion plus approfondie de la conception de la relation Élaboration chez Hobbs, en particulier.

${ }^{18}$ Voir aussi Mann et Thompson (1988) pour les relations RST 'noyau-noyau' et 'noyau-satellite', respectivement.
} 
coordonnantes, il y a une proposition commune qui peut être inférée à la fois de $\mathrm{S}^{\circ}$ et de $\mathrm{S}^{1}$; dans ce cas, lorsque les propositions inférées des deux phrases seront fusionnées, ce sera cette proposition 'superordonnée' qui sera assertée. En revanche, dans le cas des relations subordonnantes, l'une des deux propositions sera dominante; dans ce cas, ce sera la dominante qui sera assertée (et donc la dominée correspondrait de ce fait à une présupposition), une fois que les deux propositions inférées seront intégrées.

Selon cette définition, la relation Contraste sera 'subordonnante', car, intuitivement, c'est la proposition exprimée via $S^{1}$ qui est dominante; pourtant, dans le Tableau 2, il est clair que la structure sémantique de cette relation est très proche de celle de la relation Parallèle, qui est une relation coordonnante.

Par contre, la relation Élaboration est classifiée comme relevant de la catégorie coordonnante (puisqu'elle implique l'inférence d'une proposition commune à partir des deux propositions syntaxiques ou phrases à l'œuvre); cependant, intuitivement encore, la proposition 'élaborante' est dépendante vis-à-vis de la proposition 'élaborée'. La définition étendue donnée dans la cellule 2 de la $3^{\mathrm{e}}$ colonne du Tableau 2 fournirait un solide appui à cette catégorisation. Et en effet, c'est ainsi que cette relation est caractérisée par Asher et Lascarides (2003: 8). Voir aussi la conception de Bras et Le Draoulec ici-même.

Je résoudrais ce paradoxe en proposant que nous distinguions entre deux niveaux d'analyse ici (cf. aussi, Sanders, 1997): d'un côté, le niveau sémantique, et de l'autre, le niveau pragmatique, illocutoire (niveau auquel je crois que Hobbs se place en parlant de propositions dominantes ou dominées); c'est là le niveau des interventions du locuteur, le domaine de l'interaction discursive. C'est également ainsi que Roulet (2002: 148) conçoit la situation, en distinguant entre types de relations dans un discours: sémantiques, textuelles et 'praxéologiques' (structure actionnelle).

A cet égard, dans le cas de la relation Contraste, la proposition inférée de $S^{1}$ serait à égale dominance sémantiquement par rapport à celle inférée de $\mathrm{S}^{\circ}$, mais serait dominante au niveau des actes illocutoires au plan pragmatique; et dans le cas de la relation Élaboration, la proposition inférée de $S^{\circ}$ serait sémantiquement sur un pied d'égalité par rapport à celle inférée de $\mathrm{S}^{1}$ (puisqu'Élaboration, comme Contraste également, est sous-tendue par la structure qui correspond à la relation Parallèle) - mais serait dominante par rapport à cette dernière au niveau pragmatique. ${ }^{19}$

\footnotetext{
${ }^{19}$ Néanmoins, chacune des relations de cohérence retenues ici a bien un caractère sémantique, textuel ou pragmatique fondamental: Occasion, Cause-Conséquence (Résultat), Parallèle, Elaboration et Contraste seraient des relations fondamentalement 'sémantiques', car elles se fondent sur le contenu sémantique des unités reliées; Fond-Figure et Circonstance seraient 'textuelles' — puisqu'elles servent essentiellement à contextualiser l'une des deux unités en jeu, et Explication et AssertionIndice, 'pragmatiques' — vu que la relation ici implique la valeur illocutoire des unités concernées.
} 


\section{LES ÉTAPES DU TRAITEMENT D’UN MINI-TEXTE: LE RÔLE « PRO-ACTIF » DES ANAPHORIQUES}

Commençons par analyser l'exemple (3), cette fois un article de presse de fait divers tiré de notre premier corpus:

\section{'Casseurs motorisés}

$\left[\mathrm{UD}_{\mathrm{EV}}^{\circ}\right.$ Un habitant de Bressols a déposé plainte [ $\mathrm{UD}_{\mathrm{EV}}^{1}$ après avoir constaté, [ $\left[\mathrm{UD}_{\mathrm{EV}}^{2}\right.$ en rentrant chez lui, [UD ${ }_{\text {LOC-TPS }}^{3}$ mercredi en fin de journée], que sa maison avait été visitée]]]. [ $\mathrm{UD}_{\mathrm{EV}}^{4}$ Les voleurs se sont notamment intéressés au contenu du garage de l'habitation $\left[\mathrm{UD}_{\mathrm{EV}}^{5}\right.$ afin d'y dérober une moto qui y était stationnée]].' ( $L a$ Dépêche du Midi, 05/09/03)

Tout d'abord, la paire d'unités $\mathrm{UD}^{\circ}$ et $\mathrm{UD}^{1}$ serait reliée en fonction de la relation Explication (initialement, en fonction de la relation Occasion, à cause de la présence du connecteur temporel après et de la succession de deux événements: le fait de constater quelque chose (l'effraction d'une propriété) en rentrant à sa maison, préalable à l'action de déposer plainte).

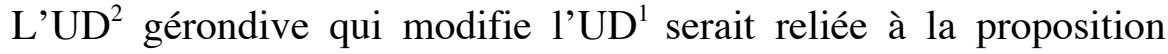
principale en fonction de la relation Circonstance, car elle fournit un repère temporel pour l'action. Nous sommes donc en présence d'une unité [UD ${ }^{\circ}$ $\left.\left[+\mathrm{UD}^{1}\left[+\mathrm{UD}^{2}\left[+\mathrm{UD}^{3}\right]\right]\right]\right]$ thétique qui rapporte l'événement 'cadre' de ce qui constitue l'essentiel de l'information. Pour sa part, la subordonnée temporelle représentée par l'unité composée $\left[\mathrm{UD}^{1}\left[+\mathrm{UD}^{2}\right]\right]$ serait reliée à

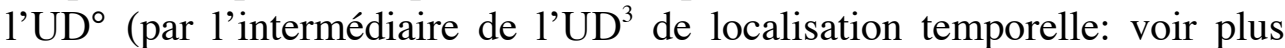
bas), car elle renvoie obligatoirement au référent du sujet de $1^{\prime} \mathrm{UD}^{\circ}-$ de par la coréférence obligatoire du sujet zéro de l'infinitive et celle (libre) des pronom lui et déterminant possessif $s a$. L' $\mathrm{UD}^{3}$ mercredi en fin de journée $e^{20}$ constitue, tout comme le SP temporel cadratif dans l'exemple (2), un acte de localisation temporelle de l'événement rapporté.

Ensuite, l'unité composée $\left[\mathrm{UD}^{4}+\mathrm{UD}^{5}\right]$ sera reliée à l'unité [UD ${ }^{\circ}$ $\left.\left[+\mathrm{UD}^{1}\left[+\mathrm{UD}^{2}\left[+\mathrm{UD}^{3}\right]\right]\right]\right]$ en fonction de la relation Élaboration, car cette unité donne des détails supplémentaires sur la situation évoquée dans l'unité initiale (à remarquer également à cet égard la présence du connecteur notamment). Le référent 'indirect' du SN lexical défini les voleurs et celui de l'anaphorique hypéronyme l'habitation en $\mathrm{UD}^{4}$, sont inférés en vertu de la proposition exprimée dans l'UD' 'que la maison du Bressolois avait été visitée'. ${ }^{21}$ L'inférence du référent 'les cambrioleurs du garage du

\footnotetext{
${ }^{20}$ Moment localisé plus précisément via la date de publication de l'édition du journal dans laquelle cet article est paru: à savoir, mercredi 3 septembre 2003.

${ }^{21}$ Nous remarquons ici le style ironique de l'auteur de ce texte - que nous observons également dans le titre - dans le caractère euphémique de l'UD' .
} 
propriétaire de la maison de Bressols' est quasi-automatique, étant donné l'existence de la variable d'argument 'Agent' disponible lexicalement grâce à l'emploi du verbe mono-transitif visiter (prédicat à deux arguments sémantiques: Agent et Locatif, respectivement), au sens de 'cambrioler'. Dans ce passif court, seul l'argument Locatif, exprimé en tant que sujet syntaxiquement obligatoire, est réalisé explicitement. De par la signification 'cambrioler' du prédicat verbal visiter ici, l'argument Agent non-exprimé en vient à désigner 'les cambrioleurs'. L'anaphore définie réalisée par les voleurs fonctionnerait de ce fait en terme d'hypéronymie, tout comme le SN l'habitation. Ces anaphores permettent donc d'accrocher l'unité [UD ${ }^{4}+$ $\left.\mathrm{UD}^{5}\right]$ à la macro-unité contextuelle $\left[\mathrm{UD}^{\circ}\left[+\mathrm{UD}^{1}\left[+\mathrm{UD}^{2}\left[+\mathrm{UD}^{3}\right]\right]\right]\right]$, car $\mathrm{UD}^{4}$ et $\mathrm{UD}^{5}$ fournissent d'autres informations sur chacun de ces référents. Le topique/cadre circonstanciel des deux unités macro est donc le même.

$\mathrm{Au}$ sein de la paire $\left[\mathrm{UD}^{4}+\mathrm{UD}^{5}\right], \mathrm{l}^{\prime} \mathrm{UD}^{5}$, par le biais du connecteur afin de, indique explicitement le But de la situation contrôlée évoquée en $\mathrm{UD}^{4}$. L'unité $\mathrm{UD}^{5}$ est également reliée à $1^{\prime} \mathrm{UD}^{4}$ en vertu du fonctionnement des deux occurrences du pronom oblique $y$ ( $y$ dérober et $y$ était stationnée) qui renvoient au référent 'le garage de l'habitation'. On voit donc très nettement ici le fonctionnement intégrateur des anaphores à l'œuvre dans ce texte (voir aussi Luscher, 1998 et Schauer, 2000 à cet égard). La Figure 2 représente de façon schématique la structure de discours associée à ce court texte:

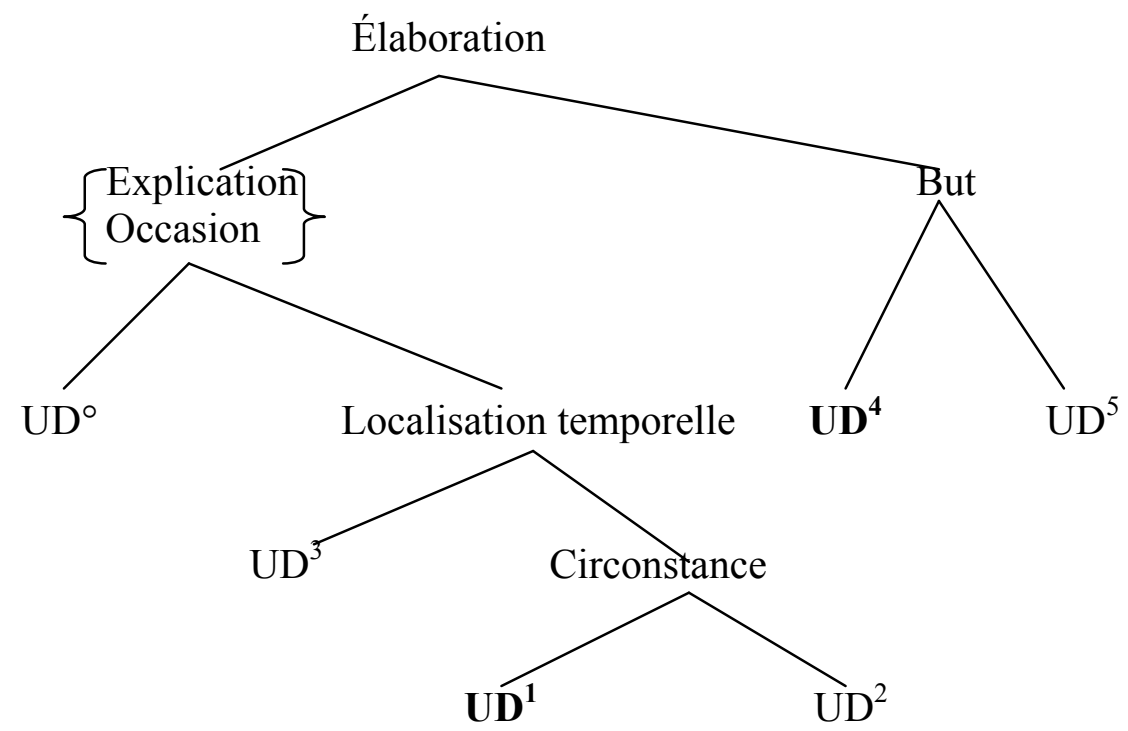

Figure 2. Structure de discours associée au texte (3), d'après le modèle de Hobbs (1990)

Cette représentation arborescente devrait se lire ainsi: procéder 'de bas en haut' (depuis les nœuds inférieurs jusqu'aux nœuds supérieurs) en se 
déplaçant d'abord de droite vers la gauche (donc vers le haut). La structure du discours à l'œuvre émergera ainsi à partir des intégrations successives des unités impliquées. Cependant, les intégrations ne procèdent pas toujours 'verticalement', depuis une unité minimale vers une unité déjà traitée à sa gauche immédiate, au plan textuel: car si une unité à droite de celle qui vient d'être traitée est accompagnée d'une unité marquée comme dépendante à son égard (une subordonnée, une incise (voir le texte (2)) ou une proposition en apposition, par exemple), alors cette intégration doit nécessairement avoir lieu en premier. Ce n'est qu'alors que cette unité composite sera intégrée avec le résultat de l'intégration en amont. Il peut également arriver qu'une intégration ultérieure rende nécessaire de réviser une interprétation antérieure - surtout si deux interprétations potentielles de cette ou de ces unité(s) avaient été possibles à ce stade.

Voici à présent un dernier exemple comportant un référent implicite, ciblé par un SN défini anaphorique:

\section{'Pyrénées-Orientales}

$\left[\mathrm{UD}^{\circ}{ }_{\mathrm{LOC}}\right.$ Le Perthus.] [UD ${ }_{\mathrm{EV}}$ Les douaniers ont saisi $59 \mathrm{~kg}$ de cocaïne dissimulés au milieu d'un chargement de luminaires]. $\left[\mathrm{UD}^{2}{ }_{\mathrm{ET}}\right.$ Le camion espagnol roulait vers la Grande Bretagne].' La Dépêche du Midi, 7/11/06, p. 8

Ici, la relation anaphorique reliant (par synecdoque avec le 'déclencheur d'antécédent') le SN défini le camion espagnol et le référent implicite évoqué via le traitement du SN un chargement de luminaires dans $1^{\prime} \mathrm{UD}^{1}$, permet à $1^{\prime} U^{2}$ d'être intégrée avec elle au moyen de la relation FondFigure (Arrière-Plan). Cette inférence est clairement de type 'connectif' plutôt que simplement 'élaboratif', selon Zwaan et Rapp (2006: 735). Si les douaniers (français) avaient contrôlé un 'chargement de luminaires' provenant d'Espagne, alors ce chargement a dû être transporté par un véhicule quelconque - le plus probable étant un camion. La situation évoquée dans les deux UD est donc la même, le temps imparfait porté par le verbe rouler dans l' $\mathrm{UD}^{2}$ signalant l'aspect inaccompli, ${ }^{22}$ et donc renvoyant à un état de choses existant au moment du traitement, et non à un nouvel événement (cf. Berthonneau et Kleiber, 1993). La destination ultime du camion (la Grande Bretagne) constitue de ce fait le contexte dans lequel l'événement principal évoqué par ce texte ('la saisine par les douaniers français des sacs de cocaïne dans le camion dont il est question') prend place.

\footnotetext{
${ }^{22}$ Plutôt un état, selon Tasmowski-de Ryck et de Mulder (1998). Voir aussi Adam (2000: 114-115).
} 


\subsection{Parallélisme entre l'invocation potentiellement multiple des relations de cohérence, et le traitement de premier et de second passage des anaphoriques}

Maintenant, s'il est vrai, comme je le soutiens, que plus d'une relation de cohérence est possible simultanément pour intégrer deux unités de discours, alors c'est la relation sémantiquement plus 'forte' qui l'emportera sur la relation plus 'faible'. Assertion-Indice ('Claim-Evidence') est sémantiquement plus forte (plus spécifique) qu'Élaboration, par exemple, puisqu'elle contient la même structure essentielle que cette dernière (voir le Tableau 2): mais pour que la relation Assertion-Indice soit applicable, la seconde des deux unités impliquées devra être interprétable comme rendant l'assertion de la première plus convaincante pour l'allocutaire ou le lecteur. Ainsi, tandis qu'Élaboration est une simple relation 'sémantique', Assertion-Indice est une relation 'pragmatique'. Les deux relations sont compatibles, car la seconde partie de la structure définitoire de chacune est dépendante par rapport à la première. Il en est de même pour les relations Occasion et Explication dans (4). En général, une relation fondamentalement 'pragmatique' l'emportera toujours sur une relation à base 'sémantique' ou 'textuelle', et une relation à base 'sémantique' primera sur une relation fondamentalement 'textuelle', là où plus d'une relation est applicable dans une situation donnée: voir à cet égard la discussion des relations coordonnante vs. subordonnante à la fin de la section 4 .

Or, ces renforcements de relations 'faibles', plus immédiatement invoquées (quasiment par défaut) pour intégrer deux unités, impliquent nécessairement un retraitement, ou un traitement de second passage, plus approfondi, de la part du compreneur. Et c'est exactement cela qui a été postulé par des psycholinguistes pour ce qui concerne la résolution en temps réel des anaphoriques de discours (cf. par exemple, Garrod et Sanford, 1999). Il y aurait à cet égard un appariement quasi immédiat d'un anaphorique arrivant, sous certaines conditions, avec un référent ayant les propriétés requises (par exemple, 'masculin', 'humain', 'singulier' pour le pronom he dans la seconde phrase du texte (1)); puis dans un second temps, une confirmation accompagnée, le cas échéant, d'une expansion de la référence initialement attribuée, ou bien une réassignation de la référence. Cette confirmation ou bien réassignation sera fonction de ce qui est prédiqué du référent de l'anaphorique (voir Kleiber, 1994: ch. 3) ainsi que de la nature de l'intégration discursive de l'unité hôte avec l'unité contextuelle pertinente. La nature de la prédication anaphorique va de toute façon sélectionner l'unité contextuelle pertinente avec laquelle elle entrera en relation.

Dans le texte (1), le pronom he sujet de la deuxième phrase va être immédiatement apparié avec le référent du SN sujet de la première (John) en vertu de l'heuristique de 'la fonction parallèle' (cf. Crawley et al., 
1990). ${ }^{23}$ Cette heuristique tend à choisir comme référent celui du SN remplissant une fonction parallèle à celle de l'anaphorique dans sa proposition; liée à cette heuristique est celle qui tend à favoriser le référent du SN sujet d'une proposition contextuelle pertinente. L'effet de ces deux heuristiques dans ce cas est de cibler le même candidat comme référent du pronom. La nature de ce qui est prédiqué du référent de he ici (à savoir, " le fait de connaître le chiffre de la combinaison du coffre-fort de Bill »), ainsi que le mode d'intégration de la seconde unité avec la première (via la relation Élaboration, dans un premier temps, puis dans un second temps, via celle d'Assertion-Indice) vont confirmer cette assignation de premier passage, en écartant définitivement toute résolution en fonction du référent 'Bill'. Comme nous l'avons vu en §2, l'appariement de he avec Bill dans le SN génitif Bill's serait déjà problématique, en tant que mise en relation textuelle.

Dans le texte (2), la résolution du SN défini le bateau au sein du SP dans la première phrase ciblera le référent implicite introduit lors de l'interprétation de l'incise temporelle $\mathrm{UD}^{2}$ lors d'une traversée maritime dans son intégration avec l'UD' ${ }^{1}$; comme dans le cas du texte (3), ce lien essentiel permettra d'intégrer ces deux unités en fonction de la relation Circonstance. De ce fait, le compreneur interprétera l'unité composite [UD ${ }^{1}$ $\left.+\mathrm{UD}^{2}\right]$ comme évoquant une situation où le propriétaire de la plantation et le capitaine sont à bord du même bateau en pleine mer au moment de l'altercation du premier avec le second. Quant au traitement de la seconde phrase de ce texte, l'heuristique de la fonction parallèle s'appliquera initialement, comme dans le traitement du texte (1), appariant le référent du pronom sujet $i l$ avec le propriétaire de la plantation évoqué lui aussi par un $\mathrm{SN}$ en position sujet de la phrase initiale. Cet appariement sera par la suite confirmé par l'intégration de l'UD ${ }^{3}$ avec l'unité composite $\left[\mathrm{UD}^{1}+\mathrm{UD}^{2}\right]$, d'abord en fonction de la relation Résultat, puis Problème-Solution, intégrations qu'il rend possibles. Comme dans le cas du texte (1), cette double intégration va définitivement écarter toute résolution en fonction des deux autres référents évoqués via des SN masculins singuliers ('le capitaine du navire' et 'le bateau sur lequel les hommes en question voyageaient').

\section{CONCLUSIONS}

$\mathrm{Du}$ point de vue du compreneur, traitant un texte en temps réel, les anaphoriques de discours seront immédiatement résolus, là où cela est possible, via des heuristiques textuelles (priorité au référent évoqué via une unité syntaxique précédente codé par le sujet de la phrase ou la proposition

23 Comme alternative, de façon équivalente, on proposera le fait que la fonction sujet attribue normalement à son référent une prééminence cognitivo-discursive par rapport aux autres référents évoqués dans la proposition - un pronom de $3^{\text {ème }}$ personne inaccentué étant surtout sensible à ce statut. 
concernée, et/ou par un SN remplissant une fonction parallèle à celle réalisée par l'anaphorique).

Cette résolution superficielle, de premier passage, sera par la suite validée par une tentative d'intégration de l'unité de discours correspondant à la prédication anaphorique avec celle qui vient d'être construite, et dont le corrélat textuel renferme l'expression référentiellement autonome ciblée par la procédure de résolution de premier passage; cette intégration sera effectuée par le biais d'une relation de cohérence appropriée, selon un appariement des arguments et des prédicats des unités concernées avec les variables spécifiées dans les structures sémantiques représentant les différentes relations (voir les définitions proposées dans les 10 cellules du Tableau 2).

Si cette intégration peut être effectuée de façon satisfaisante en fonction de l'appariement référentiel co-textuel indiqué ci-dessus, alors la résolution de premier passage est entérinée, la référence de l'anaphorique étoffée, et l'intégration via la relation de cohérence sélectionnée sera mise en œuvre. Le choix de la relation sera fonction de la relation entre ce qui est prédiqué dans les deux unités concernées, relation indiquée surtout via celle entre les deux prédicateurs (leur relation lexicale, le cas échéant, leur Aktionsart, le temps et l'aspect grammatical qu'ils portent) — puis via d'autres facettes des deux unités telles que la présence d'adverbiaux temporels ou spatiaux, la relation structuro-informationnelle existant entre les énoncés à l'œuvre, et le type de connecteur qui les relie ou qui pourrait les relier; en outre, les connaissances du monde y entrent pour une bonne part (voir à cet égard la compréhension des textes (1)-(4)).

La troisième et dernière étape consistera à insérer l'unité composite, intégrée qui constituera la sortie de ce processus d'intégration, dans le modèle mental du discours en construction. Ce modèle est situé en mémoire de travail (voir Baddeley, 1987; Cowan, 1997), avant d'être placé en mémoire à long terme pour rétention lorsqu'il sera complet.

La résolution des anaphoriques de discours a donc lieu en deux étapes distinctes: 1) initialement, et provisoirement, dès que l'anaphorique est rencontré. A ce stade, il sera résolu superficiellement, si la situation s'y prête, en fonction d'heuristiques co-textuelles (cette étape est semblable à la notion de 'bonding' ('appariement') chez Garrod \& Sanford, 1999). ${ }^{24}$ Cette première étape permettra d'ancrer les deux unités l'une à l'autre - plus particulièrement, les arguments pertinents de la seconde avec ceux de la première. 2) Puis dans un deuxième temps, au moment où une relation de cohérence est choisie, invoquée, testée, puis mise en œuvre en fonction de la

\footnotetext{
24 Les heuristiques co-textuelles que j'invoque au niveau de cette première étape ne sont pas équivalentes au 'bonding' de Garrod et Sanford (1999), pour autant: car celui-ci consiste simplement en un appariement des anaphoriques à l'œuvre avec tout $\mathrm{SN}$ se trouvant dans le co-texte environnant qui présente les mêmes valeurs de traits morpho-syntaxiques (personne, nombre et genre) - ce, quel que soit le degré de plausibilité sémantique ou pragmatique de l'appariement.
} 
construction des unités discursives correspondantes. Les anaphoriques en jeu recevront par ce biais une interprétation complète, à l'occasion étendue. Dans le cas d'exemples comme (2), (3) et (4), où le référent visé par un anaphorique n'est pas introduit co-textuellement via une expression référentiellement (semi-)autonome, les SN définis (le bateau dans (2), les voleurs dans (3) et le camion espagnol dans (4)) devront être résolus en ayant recours à la deuxième étape uniquement: le compreneur devra dans ces cas chercher ou créer (par inférence abductive) la représentation d'un référent approprié dans la structure de discours construite en amont, en invoquant directement une relation de cohérence pertinente. La première étape est utile pour permettre la vérification de l'application d'une relation de cohérence donnée, car elle assignera (provisoirement, du moins) une valeur aux variables correspondant aux positions des arguments dans les schémas généraux prévus par les définitions des différentes relations de cohérence (voir le Tableau 2).

C'est en fait la deuxième étape de ce processus qui correspondrait à l'hypothèse de Hobbs (1979), selon laquelle c'est la sélection et mise en œuvre d'une relation de cohérence pour intégrer deux unités de discours qui détermine en dernière instance la résolution des anaphoriques qu'elles peuvent comporter. Mais la mise en place de la première étape, qui permet d'ancrer les deux unités l'une à l'autre, constitue néanmoins une heuristique importante de la mise en ouvre de toute relation de cohérence. Les deux procédures sont en symbiose parfaite l'une avec l'autre - elles sont véritablement interdépendantes, l'une impliquant normalement la coprésence de l'autre.

Adresse pour correspondance:

Francis Cornish

Université de Toulouse-Le Mirail

Département Études du Monde Anglophone

5, Allée Antonio-Machado

31058 Toulouse Cedex 09

France

email:cornish@univ-tlse2.fr

\section{RÉFÉRENCES}

Adam, J-M. (2000). Le temps et les temps dans les textes. Dans: J. Moeschler et M-J. Béguelin (eds.), Référence temporelle et nominale. Actes du $3^{\text {ème }}$ cycle romand de Sciences du langage, Cluny (15-20 avril 1996), Bern etc.: Peter Lang, pp. 107-121.

Asher, N. and Lascarides, A. (2003). Logics of Conversation. Cambridge: Cambridge University Press.

Asher, N. and Vieu, L. (2005). Subordinating and coordinating discourse relations. Lingua, 115(4): 591-610. 
Baddeley, A. (1987). Working Memory. Oxford: Clarendon Press (Oxford University Press).

Berthonneau, A.-M. et Kleiber, G. (1993). Pour une nouvelle approche de l'imparfait: l'imparfait, un temps anaphorique méronomique. Langages, 112: 55-74.

Borillo, A. (1998). Les adverbes de référence temporelle comme connecteurs temporels du discours. Dans: Vogeleer et al. (eds.), pp. 131-145.

Bras, M. et Le Draoulec, A. (Ce numéro). D'abord marqueur de relation de discours subordonnante.

Charolles, M. (2005). Cohérence, pertinence et intégration conceptuelle. Dans: P. Lane (ed.), Des discours aux textes: Modèles et analyses. Rouen/Le Havre: Publications des universités de Rouen et du Havre, pp. 39-74.

Cornish, F. (2006). Relations de cohérence en discours: Critères de reconnaissance, caractérisation et articulation cohésion-cohérence. Numéro spécial de CORELA, revue en ligne, coordonné par D. Legallois: Organisation des textes et cohérence des discours. http://edel.univ-poitiers.fr/corela/document.php?id=1280.

Cornish, F. (sous presse). Inter-sentential anaphora and coherence relations in discourse: A perfect match. A paraître dans Language Sciences.

Cowan, N. (1997). Attention and Memory. An integrated framework. Oxford: Clarendon Press (Oxford University Press).

Crawley, R.A., Stevenson, R.J. and Kleinman, D. (1990). The use of heuristic strategies in the interpretation of pronouns. Journal of Psycholinguistic Research, 19(4): 245-264.

Dowty, D. R. (1986). The effects of aspectual class on the temporal structure of discourse: semantics or pragmatics? Linguistics and Philosophy, 9: 37-61.

Egg, M. and Redeker, G. (2008). Underspecified discourse representation. In: A. Benz and P. Kühnlein (eds.), Constraints in Discourse. Amsterdam/Philadelphia: John Benjamins, pp. 117-138.

Fabricius-Hansen, C., and Behrens B. (2001). Elaboration and related discourse relations viewed from an interlingual perspective. SPRIK report of the project Languages in Contrast, University of Oslo. (Disponible à http://www.hf.uio.no/german/sprik).

Garrod, S. \& Sanford, A. (1999). Ch. 1: Incrementality in discourse understanding. In: H. van Oostendorp and S.R. Goldman (eds.), The Construction of Mental Representations During Reading. Mahwah, N.J.: Lawrence Erlbaum Associates, pp. 3-27.

Hobbs, J. R. (1979). Coherence and coreference. Cognitive Science, 3: 6790. 
Hobbs, J.R. (1990). Ch. 5: The coherence and structure of discourse. In: Literature and Cognition. Leland Stanford Junior University, Calif: CSLI Lecture Notes 21, pp. 83-114.

Kehler, A. (2004). Discourse coherence. Ch.11 in L.R. Horn and G. Ward (eds.), The Handbook of Pragmatics. Oxford: Blackwell Publishing, pp. 241-265.

Kleiber, G. (1994). Anaphores et pronoms. Louvain-La-Neuve : Duculot.

Kleiber, G. et Vassiliadou, H. (Ce numéro). Sur la relation d'Élaboration: des approches intuitives aux approches formelles.

Luscher, J-M. (1998). Influence des connecteurs et des reprises pronominales sur l'interprétation d'énoncés aux temps du passé. Dans: Vogeleer et al. (eds.), pp. 113-129.

Madden, C.J. and Zwaan, R.A. (2003). How does verb aspect constrain event representations? Memory and Cognition, 31(5): 663-672.

Mann, W. C., and Thompson, S. A. (1988). Rhetorical Structure Theory: Toward a functional theory of text organization. Text, 8(3): 243-281.

Mellet, S. (1998). Présent et présentification: un problème d'aspect. Dans: Vogeleer et al. (eds.), pp. 203-213.

Murphy, M. L. (2003). Semantic Relations and the Lexicon. Cambridge: Cambridge University Press.

Prévot, L., Asher, N. et Vieu, L. (Ce numéro). Une formalisation plus précise pour une annotation moins confuse: Le cas des relations d'Arrière-Plan et d'Élaboration.

Rossari, C. (2000). Connecteurs et relations de discours: Des liens entre cognition et signification. Nancy: Presses Universitaires de Nancy.

Rothstein, S. (2004). Structuring Events. A study in the semantics of lexical aspect. Malden, USA/Oxford, UK/Carlton, Australia: Blackwell.

Roulet, E. (2002). Ch. 7: De la nécessité de distinguer des relations de discours sémantiques, textuelles et praxéologiques. Dans: H. L. Andersen et H. Nølke (eds.), Macro-syntaxe et macro-sémantique. Actes du colloque d'Arhus, 17-19 mai 2001. Berne: Peter Lang, pp. 141-165.

Sanders, T.J.M. (1997). Semantic and pragmatic sources of coherence: On the categorization of coherence relations in context. Discourse Processes, 24(1): 119-147.

Sanders, T.J.M. and Noordman, L.G.M. (2000). The role of coherence relations and their linguistic markers in text processing. Discourse Processes, 29(1): 37-60.

Schauer, H. (2000). Referential structure and coherence structure. In: Proceedings of the TALN Conference, Lausanne, 16-18 October, 2000 (http://www.coling.uni-freiburg.de/).

Smith, C. S. (2003). Modes of Discourse. The local structure of texts. Cambridge: Cambridge University Press.

Tasmowski-de Ryck, L. et De Mulder, W. (1998). L'imparfait est-il un 
temps méronomique ? Dans: Vogeleer et al. (eds.), pp. 171-189.

Vergez-Couret, M. (Ce numéro). Approche formelle du rôle discursif de l'adverbe notamment.

Vogeleer, S., Borillo, A., Vetters, C. et Vuillaume, M. (eds.). (1998). Temps et discours. Louvain-La-Neuve: Peeters.

Webber, B., Joshi, A., Stone, M. and Knott, A. (2003). Anaphora and discourse structure. Computational Linguistics, 29(4): 545-587.

Zwaan, R. A. and Rapp, D. N. (2006). Ch. 18: Discourse comprehension. In: M. T. Traxler and M. A. Gernsbacher (eds.), Handbook of Psycholinguistics. Amsterdam: Elsevier Inc., pp. 725-764. 
Address for correspondence:

Francis Cornish

Université de Toulouse-Le Mirail

Département Études du Monde Anglophone

5, Allée Antonio-Machado

31058 Toulouse Cedex 09

France

email:cornish@univ-tlse2.fr 